result in a premature decision to withdraw life support. It also prevents an impartial decision to terminate life.

The empathy that professional and family caregivers have for seriously ill patients is hard-wired into our brains. The same neural networks are stimulated in third parties reviewing evidence of a patient's suffering. This neurologic mechanism makes us want to end suffering, even if it means terminating life. However, it is the same mechanism that prevents us from making the truly impartial decision necessary to terminate life. An innate understanding of this dilemma is the basis for our customary prohibition on terminating life. It is a dilemma that we may never be able to solve.
Vivian C. McAlister, MD

Coeditor, Canadian fournal of Surgery

Competing interests: None declared.

DOI: $10.1503 /$ cjs. 002616

\section{References}

1. McAlister V. Maimonides's cooling period and organ retrieval. Can 7 Surg 2004:47:8-9.

2. ALS Society of Canada. Compassion fatigue. Available: http://als .ca/sites/default/files/files/Compassion_Fatigue\%281\%29.pdf (accessed 2016 Feb. 25).

\title{
La fatigue des aidants et la prise de décisions en fin de vie par autrui
}

Les opinions exprimées dans cet éditorial sont celles de l'auteur et ne reflètent pas nécessairement la position de l'éditeur.

La décision de la Cour suprême du Canada dans l'affaire Carter c. Canada (2015 CSC 5) concerne le droit d'un adulte capable de décision à recourir à l'aide médicale à mourir si ses souffrances, dues à un problème de santé grave et irrémédiable, lui sont intolérables. En plus d'avoir élargi les paramètres pour inclure les mineurs capables de décision et les directives préalables, les discussions sur la décision pourraient aussi avoir influencé notre vision d'un scénario de fin de vie distinct mais relié : le retrait du maintien des fonctions vitales. Le nombre d'agents anesthésiants utilisés pour soulager un patient durant le retrait semble d'ailleurs avoir augmenté récemment.

Comment concilier le fait que, d'une part, toutes nos traditions juridiques et religieuses supposent que l'aversion à mettre fin à la vie est intrinsèque à la civilisation et que, d'autre part, un groupe de citoyens empathiques croit que l'aide à mourir pourrait avoir un rôle dans les soins aux personnes gravement malades? Une décision de mettre fin à la vie d'un patient peut-elle être impartiale?

La situation clinique décrite dans la décision survient rarement dans le continuum des scénarios de fin de vie. La vaste majorité des patients ne sont pas en mesure de communiquer leur consentement à mourir, et la plupart désignent leur partenaire de vie pour prendre des décisions essentielles à leur place lorsqu'ils en seront incapables. Même si les patients espèrent guider leurs proches en leur faisant connaître leurs volontés à l'avance, chaque décision doit être prise dans un contexte unique, et c'est sur les épaules de ces délégués que reposera la pleine responsabilité du choix.

Bien que la plupart des patients préfèrent que leur plus proche parent prenne les décisions à leur place, les avocats recommandent de faire appel à un tiers qui ne profitera ni financièrement ni autrement du décès du patient. Par ailleurs, il ne serait pas acceptable de confier ces décisions à l'État ou à un comité d'hôpital en raison des erreurs survenues dans le passé, notamment la stérilisation forcée et les programmes d'euthanasie instaurés sous le régime nazi. Cependant, même un tiers pourrait être incapable de rester impartial.

Les protocoles de fin de vie exigeraient probablement l'intervention de 2 médecins pour cerner les problèmes médicaux. Cette redondance confirme la nature subjective de ces questions et le risque de conflit d'intérêts. Utilisée pour la première fois en France pour rendre possibles les autopsies moins de 24 heures après le décès, la stratégie du recours à 2 médecins a ensuite été appliquée à la décision d'interner des patients pour un traitement psychiatrique et à la déclaration de mort cérébrale aux fins de transplantation ${ }^{1}$. Le médecin du patient devrait-il compter parmi les décideurs? Bien que leur opinion risque peu d'être influencée par la perte ou le gain d'argent, les médecins peuvent partager avec les représentants des patients un autre type de conflit d'intérêts : le risque de fatigue des aidants.

On a déjà démontré que les réseaux de neurones stimulés par la peur et la douleur sont aussi activés par l'observation, ou même l'imagination, d'événements qui 
causent ces réponses. Les aidants souffrent avec ceux dont ils prennent soin. Les proches qui vivent par procuration la douleur et la souffrance causées par des soins ultimement futiles ont de la difficulté à résister à la pression. Le désir d'empêcher que la souffrance se reproduise est naturel. Les chirurgiens essaient d'en tirer des leçons afin d'améliorer les soins qu'ils donneront à leurs futurs patients et les résultats qu'ils obtiendront. Pour d'autres, l'interruption de la vie à cette étape peut être considérée comme une option préférable pour prévenir des souffrances inutiles. Pour certaines maladies, comme la sclérose latérale amyotrophique (SLA) avancée, les perspectives de progrès semblent éloignées, ce qui renforce le désir de mettre fin « avec dignité » à une phase qui ne permet aucun espoir.

Le stress lié aux soins et l'exposition prolongée à la souffrance ont des répercussions sur les aidants familiaux et professionnels. On a recensé plusieurs formes de fatigue des aidants, mais elles sont mal définies. La Société canadienne de la SLA croit que la fatigue de compassion survient quand les aidants commencent à ressentir la douleur et la souffrance du patient et perdent de vue leur identité et leur rôle dans les soins². L'organisme a répertorié 16 symptômes : certains ressemblent à ceux de la dépression, comme la difficulté à se concentrer ou à dormir; d'autres se rapprochent de ceux de la dépendance, comme l'obsession ou les problèmes au travail. L'irritabilité et la difficulté à prendre des décisions sont des traits distinctifs de la fatigue de compassion.

La fatigue des aidants peut être aggravée par le maelstrom des interventions salvatrices suivies de complications et des inévitables obstacles qui précèdent le rétablissement. La plupart des chirurgiens ont vu des familles alterner entre le désir de tout tenter et le désespoir face à tout traitement, et ce, en quelques heures ou quelques jours. Les chirurgiens qui ont multiplié les efforts pour sauver un patient risquent la même confusion émotionnelle. Pour accroître leur résilience, ils doivent reconnaître les signaux d'alarme et chercher l'aide d'un collègue pour soigner le patient. Par ailleurs, la tendance moderne à la rotation des équipes de soins limite le soutien que peut offrir un chirurgien aux familles. Tous ces facteurs risquent de mener à un retrait prématuré du maintien des fonctions vitales. Ils mettent aussi en jeu l'impartialité d'une décision concernant l'interruption de la vie.

L'empathie qu'éprouvent les aidants professionnels et familiaux pour les patients gravement malades est bien ancrée dans nos cerveaux. Les mêmes réseaux de neurones sont stimulés chez des tiers qui étudient le cas d'un patient souffrant. Ce mécanisme neurologique nous pousse à vouloir mettre fin à la souffrance, même s'il faut abréger la vie. Cependant, ce même mécanisme nous empêche de faire preuve de l'impartialité nécessaire pour prendre la décision de mettre fin à une vie. C'est une compréhension innée de ce dilemme qui nous pousse conventionnellement à interdire de mettre fin à la vie. Voilà un dilemme qu'il ne nous sera peutêtre jamais donné de résoudre.

\section{Vivian C. McAlister, MD}

Corédacteur, Fournal canadien de chirurgie

Intérêts concurrents: Aucun déclaré.

DOI: $10.1503 /$ cjs.003616

\section{Références}

1. McAlister V. Maimonides's cooling period and organ retrieval. Can 7 Surg 2004;47:8-9.

2. ALS Society of Canada. Compassion fatigue. Disponible : http://als .ca/sites/default/files/files/Compassion_Fatigue\%281\%29.pdf (consulté 2016, 25 février). 